PAPER

\title{
Gross morphology and morphometric sequelae in the hippocampus, fornix, and corpus callosum of patients with severe non-missile traumatic brain injury without macroscopically detectable lesions: a T1 weighted MRI study
}

\author{
F Tomaiuolo, G A Carlesimo, M Di Paola, M Petrides, F Fera, R Bonanni, R Formisano, \\ P Pasqualetti, C Caltagirone
}

J Neurol Neurosurg Psychiatry 2004;75:1314-1322. doi: 10.1136/jnnp.2003.017046

See end of article for authors' affiliations

Correspondence to:

Dr F Tomaivolo, IRCCS

'Santa Lucia', Via

Ardeatina 309, 00179

Roma, Italy; tomaivolo@

hsantalucia.it

Received 16 April 2003

Revised 6 December 2003

Accepted

7 December 2003

\begin{abstract}
Objective: The gross morphology and morphometry of the hippocampus, fornix, and corpus callosum in patients with severe non-missile traumatic brain injury (nmTBI) without obvious neuroradiological lesions was examined and the volumes of these structures were correlated with performance on memory tests. In addition, the predictability of the length of coma from the selected anatomical volumes was examined. Method: High spatial resolution T1 weighted MRI scans of the brain $\left(1 \mathrm{~mm}^{3}\right)$ and neuropsychological evaluations with standardised tests were performed at least 3 months after trauma in 19 patients.

Results: In comparison with control subjects matched in terms of gender and age, volume reduction in the hippocampus, fornix, and corpus callosum of the nmTBI patients was quantitatively significant. The length of coma correlated with the volume reduction in the corpus callosum. Immediate free recall of word lists correlated with the volume of the fornix and the corpus callosum. Delayed recall of word lists and immediate recall of the Rey figure both correlated with the volume of the fornix. Delayed recall of the Rey figure correlated with the volume of the fornix and the right hippocampus.

Conclusion: These findings demonstrate that in severe $\mathrm{nmTBI}$ without obvious neuroradiological lesions there is a clear hippocampal, fornix, and callosal volume reduction. The length of coma predicts the callosal volume reduction, which could be considered a marker of the severity of axonal loss. A few memory test scores correlated with the volumes of the selected anatomical structures. This relationship with memory performance may reflect the diffuse nature of the damage, leading to the disruption of neural circuits at multiple levels and the progressive neural degeneration occurring in TBI.
\end{abstract}

$\mathrm{N}$ on-missile traumatic brain injury (nmTBI) may result in focal damage of the brain parenchyma, owing to cerebral contusion, intracranial haematoma, or high intracranial pressure. It may also result in diffuse brain damage, diffuse axonal injury, hypoxic brain damage, brain swelling, and diffuse vascular injury. ${ }^{1}$ Diffuse brain damage is suspected in an unconscious patient who does not exhibit an obvious lesion using conventional computerised tomography or MRI. ${ }^{2-4}$ However, quantitative studies of brain MRIs collected at least 6 weeks post-injury have shown that moderate to severe traumatic brain injury results in decreases in the volume of the hippocampus, ${ }^{5-8}$ fornix, ${ }^{8-10}$ and corpus callosum. ${ }^{10-12}$

Variable results have been obtained in MRI studies evaluating the relationship between length of coma and the callosal volume reduction. ${ }^{10-13}$ In addition, investigations of the relationships between memory impairment, one of the most frequent cognitive sequelae of traumatic brain injury, ${ }^{14}$ with anatomical volumes of the hippocampus and the fornix have yielded inconsistent results..$^{5-10}$

It could be argued that inconsistent results in the relationship between volume changes in specific anatomical structures and the behavioural consequences of traumatic brain injury could have arisen because of heterogenous cohorts. Focal brain lesions in some patients might have been responsible for impairment on specific cognitive tests, thus confounding the evaluation of the relationship between cognitive dysfunction and the volume changes in selected brain structures. Indeed, follow up studies have revealed different neuropsychological performance patterns when comparing diffuse versus focal brain lesions in traumatic brain injured patients. ${ }^{15}$ By selecting a sample of nmTBI patients without large radiological lesions, it should be possible to estimate the volume modifications of specific anatomical structures and their relationship with coma and memory impairment, while avoiding the confounding effect induced by large brain lesions.

Another possible source of the lack of a consistent relationship between neuroanatomical volumes and behavioural data may be the different methods used in macroscopic neuroanatomical studies to normalise the brain measures. In order to reduce the inter-individual variability in brain size, some studies used forebrain volume, cranial capacity, or cross sectional cerebral area to derive a ratio measure of the structures of interest, ${ }^{5} 69101718$ while other studies used the above measures as covariates in statistical analysis. ${ }^{19} 20$ Recently, Bermudez and Zatorre ${ }^{21}$ tested different methods of normalising corpus callosum volume measurements and have concluded that a reliable measure could be obtained by registering the MRI brain volumes into the Talairach proportional stereotaxic space using the algorithm

Abbreviations: $n m T B I$, non-missile traumatic brain injury; $R O I$, region of interest 
developed by Collins et al. ${ }^{22}$ In the present study, we applied this method of normalisation for callosal, hippocampal, and fornix volume measurements.

Patients who had experienced a severe nmTBI at least 3 months before the present MRI study and who did not have large detectable brain lesions were investigated. The aim of the present study was fourfold: (a) to examine the gross morphology of the hippocampus, fornix, and corpus callosum; $(b)$ to estimate the volume reductions in the hippocampus, fornix, and corpus callosum after normalising the brains in Talairach space; (c) to investigate whether the length of coma can predict the extent of volume reduction in any one of the neuroanatomical structures considered; and (d) to examine whether the volumes of the structures of interest correlate with performance on standard neuropsychological tests of memory.

\section{Methods}

Nineteen patients ( 12 males, 7 females; mean (SD) age 28.25 (13.05) and 42.71 (16.37) years; range 17-60 and 19-68 years, respectively), who had experienced a severe nmTBI (Glasgow Coma Scale <8) and had recovered from a period of coma that ranged in duration from 3 to 60 days, were studied. They were selected from a consecutive sample of 28 nmTBI patients who were attending the rehabilitation programme in the Santa Lucia Hospital in Rome. Patients who had severe language or attention deficits that prevented neuropsychological testing were excluded from the study. Patients who exhibited detectable lesions that were larger than $1.6 \mathrm{~cm}^{3}$ - that is, approximately $1 / 1000$ of the overall brain volume in standardised stereotaxic space as seen on the high spatial resolution MRI collected at the time of the study, were also excluded (see table 1 for details of the lesions). The decision to define this as the small lesion was reached by consensus among the investigators. The injury of the patients occurred 3-113 months (mean (SD) 20.16 (28.9)) before the time of testing. Nineteen gender matched control subjects who were comparable in age were also studied (12 males, 7 females; mean (SD) age 30.08 (13.15) and 44.71 (17.21) years; range 18-60 and 23-72 years, respectively). Neither the nmTBI nor the control subjects had a history of neurological or psychiatric disorder or drug abuse. All participants gave informed consent. The protocol was approved by the research ethics committee of the Santa Lucia Foundation.

\section{MRI}

Magnetisation prepared rapid gradient echo Tl weighted images (Erlangen, Germany; $1 \mathrm{~mm}$ isotropic voxel, repetition time $11.4 \mathrm{~ms}$, echo time $4.4 \mathrm{~ms}$, flip angle $=15^{\circ}$ ) were obtained with a Siemens 1.5T Vision Magnetom MR system. The high spatial resolution brain sampling of $1 \mathrm{~mm}^{3}$ allowed images to be rotated by small angles in the three orthogonal planes, thus facilitating the identification of anatomical landmarks for the selection of the regions of interest. MRI volumes were rotated so that the septum pellucidum and at least a large part of the falx would be visible in the sagittal plane. This is particularly important for obtaining a reliable measure of the corpus callosum as it is sampled at its most medial part. In addition, to better visualise the hippocampus, each MRI volume was manually oriented along the main axis of the hippocampus (see fig l).

\section{Regions of interest: anatomical landmarks and boundaries \\ Hippocampal volume}

The axial view was the default view, but reference to the coronal and sagittal views was also made when identifying
Table 1 Site and volume of brain lesions

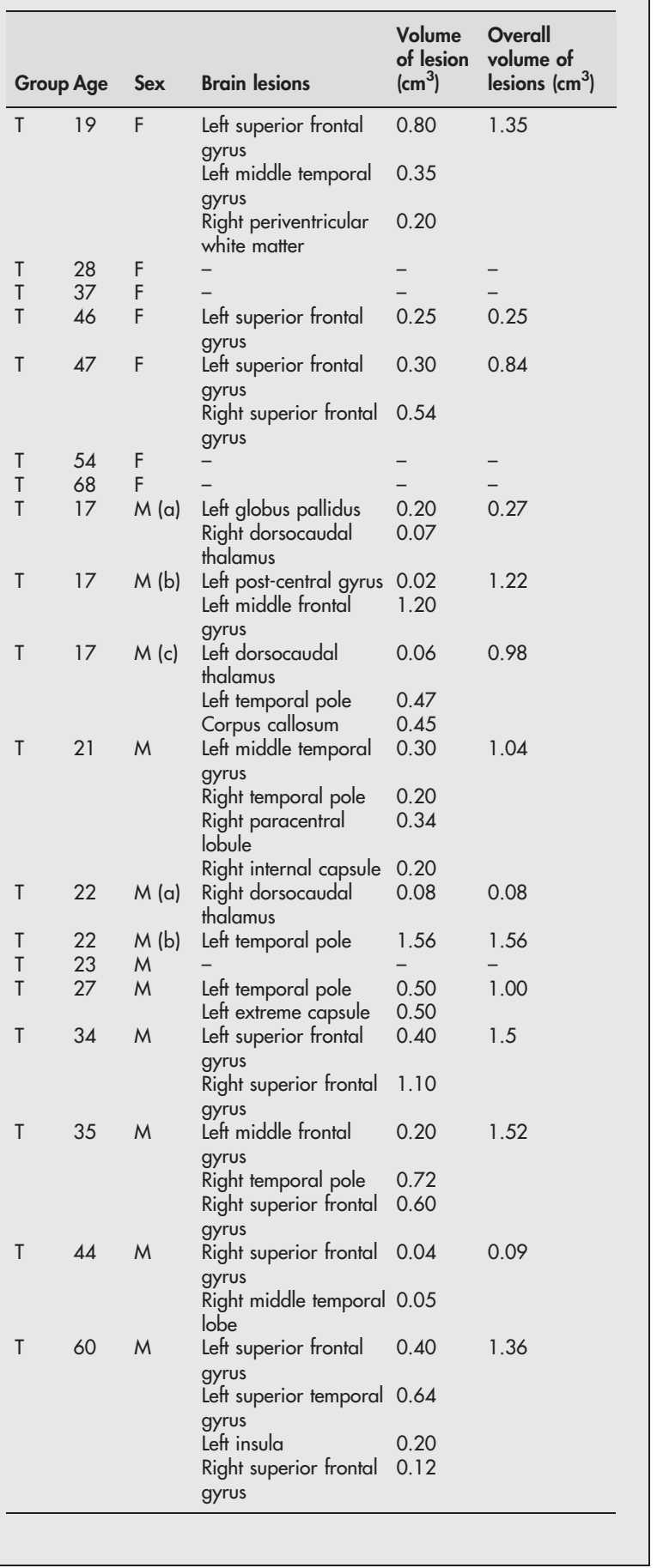

the boundaries of the hippocampal region of interest (ROI). The subiculum was excluded from the definition of the hippocampal volume. The anatomical landmarks selected to define the hippocampal volume were: rostro-laterally, the temporal horn of the lateral ventricle; caudally, the section immediately before the most posterior part of the tail of the hippocampus-that is, the point where an ovoid mass of gray matter started to appear inferomedial to the trigone of the lateral ventricle; medially, the sulcus hippocampi, excluding the subiculum and probably a small part of the cornu ammonis. Thus, the hippocampal volume as measured in the present study represented the dentate gyrus and the cornu 


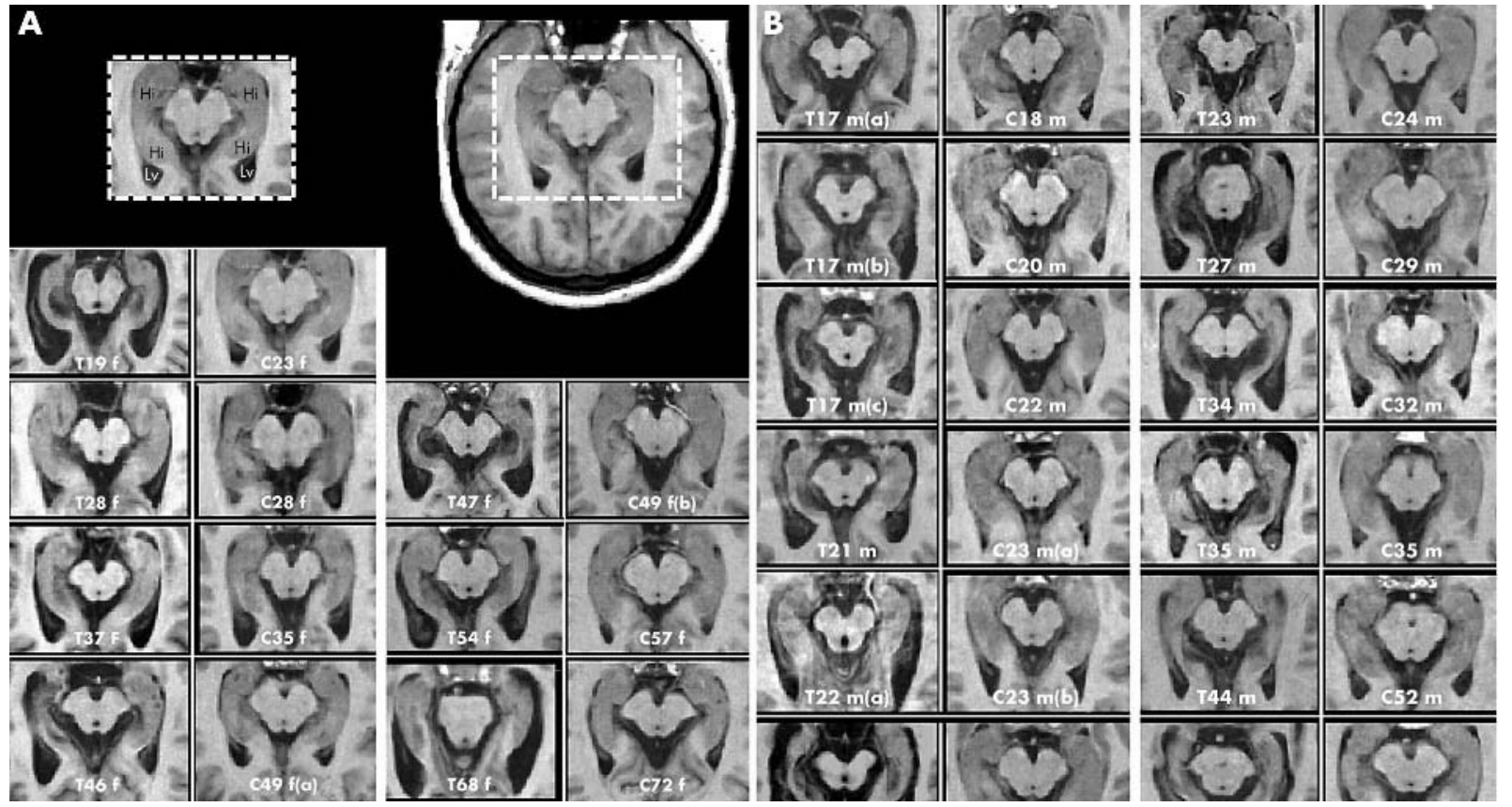

Figure 1 The hippocampal region of the nmTBls is shown in the left column ( $T$, trauma patients) and in the right column that of the control subjects (C, control subjects) matched for gender and age. The numbers indicate the age while the letters ' $f$ ' and ' $m$ ' refer to female and male subjects, respectively. The dashed rectangle indicates the region of the brain from which the hippocampal images were selected (A). As can be seen from $(A)$ and $(B)$, the hippocampal volume reduction was evident compared with their matched control in all the nmTBI patients, except for nmTBI Tm23. Hi, hippocampus; $\mathrm{LV}$, lateral ventricle.

ammonis, but not the alveus. The latter was defined as the white band observed at the top of the cornu ammonis.

\section{Fornix volume}

The coronal view was the default view, but the axial and sagittal views were also used to identify the boundaries of the fornix. The anatomical landmarks selected to define the volume of the fornix were the following. Starting from the left and right crura of the fornix, we proceeded rostrally and caudally as far as the corpus callosum. The limit of the body of the fornix with the septum pellucidum was followed as far as the anterior commissure.

\section{Corpus callosum volume}

This was measured from the mid-sagittal MRI slice ( $1 \mathrm{~mm}$ thickness) at the point where the septum pellucidum and the falx were simultaneously visible. The boundaries were, dorsally and rostrally, the pericallosal sulcus, and ventrally, the III ventricle and the cisterna superior. When it was not possible to distinguish between the corpus callosum and the fornix, a boundary was chosen by drawing the shortest line between the anterior and the posterior end of the corpus callosum with the fornix.

\section{Labelling of the region of interest}

The ROI was mapped using the interactive program Display ( J D McDonald, Brain Imaging Centre, Montreal Neurological Institute; www.bic.mni.mcgill.ca/software/display). This program permits labelling of voxel regions on each slice of the MRI volume and allows for the simultaneous visualisation in three dimensions of the movement of the cursor in the sagittal, axial, and coronal planes of the MRI. Any area can be selected and, by using the Display "mouse brush", the voxels of the area can be coloured. This colouring procedure, accompanied with the $3 \mathrm{D}$ view of the MRI planes, allows better identification of the ROI. In this study, the ROIs were identified by their landmarks and then the voxels of the area were marked by colouring. Once the ROIs had been thus selected, Display calculated the volume. A similar procedure had been used previously. ${ }^{23-25}$ The raters were blinded to the clinical information. The inter-rater reproducibility (evaluated by means of intra-class correlation coefficient were: hippocampi $=0.85$ (raters were FF and FT), corpus callosum $=0.90$ (raters were MDP and FT), and fornix $=0.91$ (intra-rater MDP). Each ROI was subsequently re-drawn by mutual consent among the raters (MDP and FT), and this measure was used for statistical analysis.

\section{Brain volume normalisation}

In order to reduce the inter-individual variability in gross brain size, each of the MRI brain volumes from which the volumetric measures were collected was registered into the Talairach proportional stereotaxic space, using a 9 parameter registration algorithm. ${ }^{22}$ The normalised Talairach stereotaxic space ROI measures were automatically obtained by applying the appropriate dimension of scaling recovered during the Talairach stereotaxic brain volume transformation. A similar procedure has been used in previous studies. ${ }^{21} 26$

\section{Focal lesion measurement}

We defined as lesion the missing brain tissue that was visually detectable on the high-spatial resolution Tl weighted MRI images. Each visually detected lesion was drawn by two raters (MDP and FT) following mutual consent, using the labelling method described in the ROI procedure.

\section{End of coma}

The end of coma was defined as the date when the patient was able to execute a simple command, which was retrospectively established based on the anamnestic report. 


\section{Neuropsychological testing}

This testing was carried out within a period of 2 weeks from the MRI scan. Overall estimation of the global intellectual and mnestic performance of the patients was obtained by administering the Wechsler Adult Verbal Intelligence Scale Revised $^{27}$ and the Wechsler Memory Scale. ${ }^{28}$ More detailed neuropsychological testing was focused on memory and included the tests described below. The test scores were adjusted for age, years of education, and gender. For each one of the following tests, the adjusted performance score falling below the lower limit of the $95 \%$ tolerance interval of the normal population was considered as pathological. ${ }^{29-31}$

\section{Word list learning ${ }^{30}$}

The examiner reads a list of 15 semantically unrelated words at the rate of one word every 2 seconds and the subject is required to repeat, immediately afterwards, as many words as possible. The test consists of five consecutive such immediate free recall trials, followed by a delayed recall test that is administered 15 minutes after the fifth immediate free recall. Performance scores are the number of words correctly recalled (pathological score $<28.33$ and 4.69, maximum score 75 and 15 for the immediate and delayed tests, respectively ${ }^{30}$ ).

\section{Immediate and delayed recall of a short story ${ }^{31}$}

The examiner reads a short story that the subject must recall immediately after presentation and also after a 15 minute interval. Performance scores are defined as the number of informative units reported (pathological score $<3.10$ and 2.39, maximum score 6 and 6 for the immediate and delayed tests, respectively ${ }^{31}$ ).

Rey-Osterrieth complex figure test $t^{31} 32$

The test consists of a freehand copy of a complex, nonverbalisable figure that is followed by an immediate and a delayed (20 minutes later) reproduction from memory. Performance scores are defined as the number of elements that are correctly reproduced (pathological score $<6.44$ and 6.33, maximum score 36 and 36 for the immediate and delayed tests, respectively ${ }^{31}$ ).
The scores on the memory tests for each subject are presented in table 2 .

\section{Statistical analysis}

Before applying parametric analysis, Kolmogorov-Smirnov and Shapiro-Wilk tests were applied to identify deviations from 'normality' for all the examined anatomical and neuropsychological distributions of scores. Only the data from the word list learning delayed recall test (KolmogorovSmirnov statistic $=0.220, \mathrm{df}=19, \mathrm{p}=-0.016$; Shapiro-Wilk statistic $=0.85, \quad \mathrm{df}=19, \mathrm{p}=0.011)$ deviated significantly from the normal distribution.

As it has been demonstrated that there are age and gender related differences in the volume of the anatomical structures of interest, ${ }^{33}{ }^{34}$ we carried out a regression analysis of age with left and right hippocampus, corpus callosum, and fornix volumes in the control group. The regression analysis revealed no significant correlation between age and volumes of the left hippocampus ( $\mathrm{r}=0.106, \mathrm{NS})$, right hippocampus $(\mathrm{r}=0.248$, NS), or corpus callosum $(\mathrm{r}=0.096, \mathrm{NS})$, but the correlation with the volume of the fornix was significant $(\mathrm{r}=0.46, \mathrm{p}=0.047)$. To test for gender effect, an unpaired $t$ test comparing male versus female for each anatomical measure was applied to the data from the control group. There were no significant differences between male and female for the volume of the left hippocampus $\left(\mathrm{t}_{(17)}=0.827\right.$, $\mathrm{NS})$, the right hippocampus $\left(\mathrm{t}_{(17)}=0.495, \mathrm{NS}\right)$, the fornix $\left(\mathrm{t}_{(17)}=0.69, \mathrm{NS}\right)$, or the corpus callosum $\left(\mathrm{t}_{(17)}=1.42\right.$, NS $)$.

Finally, it has been shown that cell loss after non missile head injury occurs continuously over a considerable period of time. ${ }^{35}$ For this reason, the temporal interval between head injury and test was correlated with the investigated anatomical volumes. There was no significant correlation of the anatomical volumes and the temporal interval between head injury and test (left hippocampus, $\mathrm{r}=0.36$, NS; right hippocampus $\mathrm{r}=0.22$, NS; corpus callosum $\mathrm{r}=-0.36$, NS; fornix $r=-0.188$, NS). A linear anatomical reduction was assumed in the above correlation analyses.

The following data analyses were carried out: (1) A mixed two way analysis of variance on hippocampal volumes, the inter-subjects factor being "group" (nmTBIs versus controls) and the intra-subjects factor being "hemisphere" (left versus

Table 2 Behavioural data

\begin{tabular}{|c|c|c|c|c|c|c|c|c|c|c|c|c|c|}
\hline Age & Sex & $\begin{array}{l}\text { Coma } \\
\text { length } \\
\text { (days) }\end{array}$ & $\begin{array}{l}\text { Head } \\
\text { iniury to } \\
\text { time test } \\
\text { interval } \\
\text { (months) }\end{array}$ & Literacy & $\begin{array}{l}\text { WAIS } \\
\text { VIQ }\end{array}$ & $M Q$ & $\mathrm{VIQ}-\mathrm{MQ}$ & $\begin{array}{l}\text { Words } \\
\text { immediate } \\
\text { recall (cut } \\
\text { off }=28.53 \text { ) }\end{array}$ & $\begin{array}{l}\text { Words delayed } \\
\text { recall (cut } \\
\text { off }=4.69 \text { ) }\end{array}$ & $\begin{array}{l}\text { Short story } \\
\text { immediate } \\
\text { recall (cut } \\
\text { off }=3.10 \text { ) }\end{array}$ & $\begin{array}{l}\text { Short story } \\
\text { delayed } \\
\text { recall (cut } \\
\text { off }=2.39 \text { ) }\end{array}$ & $\begin{array}{l}\text { Rey figure } \\
\text { immediate } \\
\text { recall (cut } \\
\text { off }=6.44 \text { ) }\end{array}$ & $\begin{array}{l}\text { Rey figure } \\
\text { delayed recall } \\
\text { (cut off }=6.33 \text { ) }\end{array}$ \\
\hline 19 & $\mathrm{~F}$ & 30 & 10 & 11 & 71 & 49 & 22 & $12.1^{*}$ & $0.0^{*}$ & $0.0^{*}$ & $0.0^{*}$ & - & - \\
\hline 28 & $\mathrm{~F}$ & 60 & 113 & 13 & 90 & 72 & 18 & $16.3^{*}$ & $2.2^{*}$ & $2.9^{*}$ & 2.6 & 8.4 & 8.1 \\
\hline 37 & $\mathrm{~F}$ & 10 & 12 & 18 & 113 & 97 & 16 & $23.7^{*}$ & 5.2 & 6.5 & 7.3 & $0.1^{*}$ & $0.8^{*}$ \\
\hline 46 & $\mathrm{~F}$ & 13 & 8 & 18 & 87 & 80 & 7 & $14.3^{*}$ & $1.1^{*}$ & 3.2 & 2.6 & $4.6^{*}$ & $4.3^{*}$ \\
\hline 47 & $\mathrm{~F}$ & 45 & 11 & 18 & 98 & 107 & -9 & 29.7 & $4.5^{\star}$ & 4.8 & 4.4 & $4.6^{*}$ & $2.8^{*}$ \\
\hline 54 & $\mathrm{~F}$ & 3 & 10 & 13 & 105 & 92 & 13 & 36.0 & $4.5^{*}$ & 4.6 & $0.0^{*}$ & 8.7 & 12.0 \\
\hline 68 & $\mathrm{~F}$ & 3 & 3 & 5 & 83 & 66 & 17 & 29.0 & 5.4 & 4.2 & $1.7^{*}$ & 10.0 & 9.8 \\
\hline 17 & $M(a)$ & 43 & 13 & 10 & 91 & 69 & 22 & $8.9^{*}$ & $0.0^{*}$ & $2.8^{*}$ & $0.0^{*}$ & $4.9^{*}$ & $4.8^{*}$ \\
\hline 17 & $M(b)$ & 15 & 6 & 10 & 106 & 114 & -8 & 30.9 & 6.2 & 5.3 & 2.6 & 9.9 & $0.0^{*}$ \\
\hline 17 & $M(c)$ & 40 & 11 & 10 & 96 & 84 & 12 & $22.9^{*}$ & $3.2^{*}$ & - & - & 12.9 & 11.8 \\
\hline 21 & $M$ & 39 & 11 & 9 & 79 & 60 & 19 & $20.9^{*}$ & $2.2^{*}$ & 4.5 & 4.4 & - & - \\
\hline 22 & $M(a)$ & 30 & 11 & 13 & 82 & 57 & 25 & $13.1^{*}$ & $0.0^{*}$ & 3.8 & $0.0^{*}$ & $0.0^{*}$ & $0.0^{*}$ \\
\hline 22 & $M(b)$ & 55 & 78 & 8 & 77 & 59 & 18 & $8.9^{*}$ & $0.0^{*}$ & $0.0^{*}$ & $0.0^{*}$ & $0.0^{*}$ & $0.0^{*}$ \\
\hline 23 & $M$ & 41 & 8 & 8 & 89 & 86 & 3 & 29.9 & $4.5^{\star}$ & 3.9 & 6.9 & $0.0^{*}$ & $0.0^{*}$ \\
\hline 27 & $M$ & 28 & 11 & 13 & 87 & 67 & 20 & $12.2^{*}$ & $0.0^{*}$ & - & - & $1.6^{*}$ & $0.0^{*}$ \\
\hline 34 & $M$ & 28 & 50 & 18 & 110 & 90 & 20 & $16.7^{*}$ & $0.0^{*}$ & $1.6^{*}$ & 4.5 & $3.6^{*}$ & - \\
\hline 35 & $M$ & 18 & 3 & 13 & 111 & 90 & 21 & $21.5^{*}$ & $3.6^{*}$ & 7.4 & 6.1 & $5.8^{*}$ & $2.2^{*}$ \\
\hline 44 & $M$ & 8 & 5 & 8 & 88 & 67 & 21 & $23.8^{*}$ & $0.0^{*}$ & 3.1 & $0.8^{*}$ & $4.1^{*}$ & $1.1^{*}$ \\
\hline 60 & $M$ & 45 & 9 & 5 & 91 & 69 & 22 & $20.4^{*}$ & $2.2^{*}$ & 4.0 & $0.0^{*}$ & $1.9^{*}$ & $1.6^{*}$ \\
\hline
\end{tabular}

*Pathological score.

WAIS VIQ, Wechsler Adult Intelligence Scale - Verbal Intelligence Quotient; MQ, Memory Quotient; VIQ-MQ, Verbal Intelligence Quotient minus Memory Quotient. 


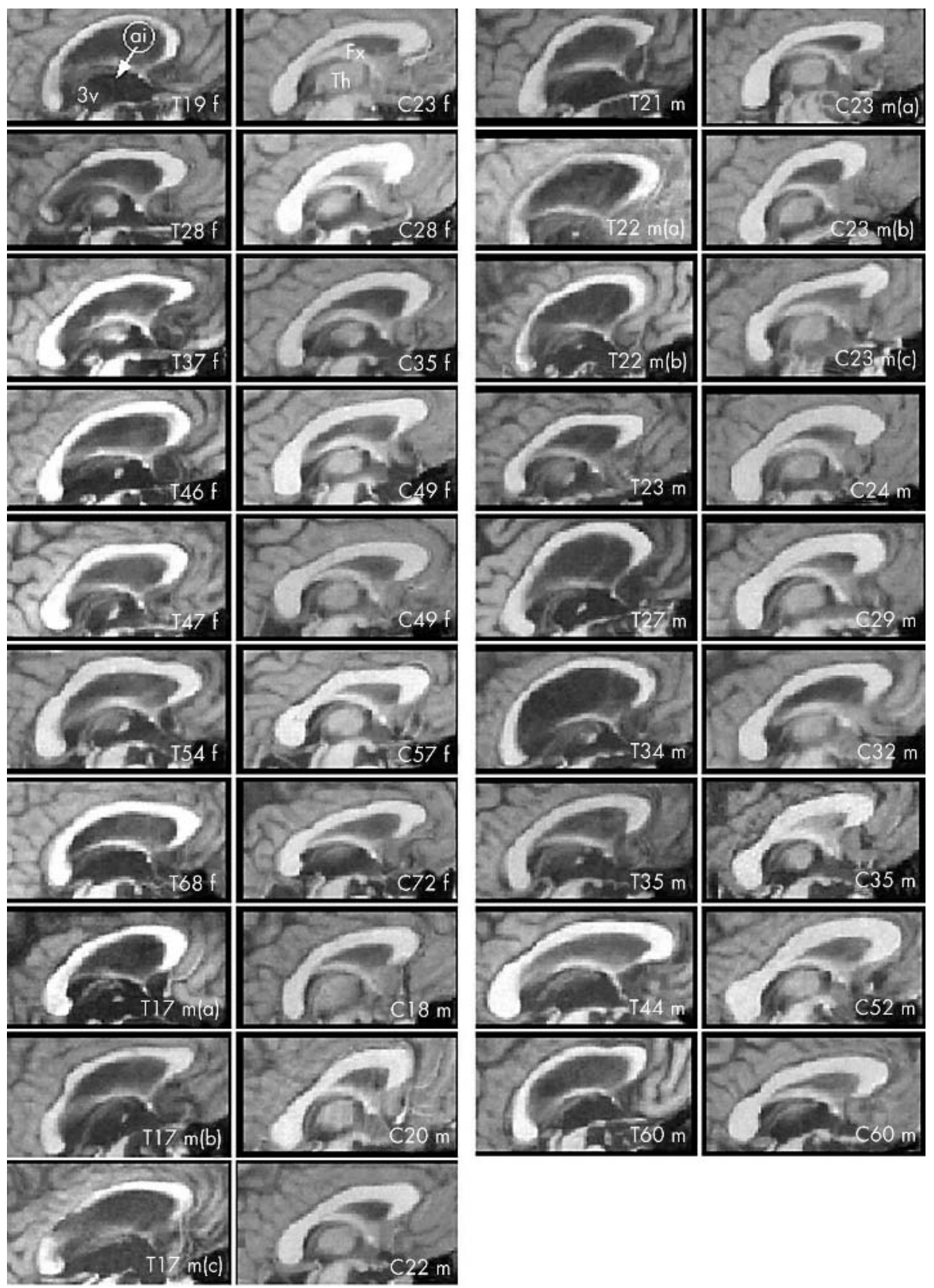

Figure 2 The morphology of the corpus callosum as visualised from the sagittal view: right column for the nmTBls ( $T$, trauma patients) and left column for the control subjects $(C$, control subjects) matched for gender and age. The numbers indicate the age, and the letters ' $f$ ' and ' $m$ ' refer to female and male subjects, respectively. The corpus callosum in nmTBls is reduced compared with that of the control subjects. The shape of the corpus callosum in the nmTBls appears to be more concave than that of the normal subjects. Additionally, the fornix appears very thin in the nmTBls. Note that the III ventricle appears to have widened and only the adesio interthalamica is sometimes visible, whereas in control subjects the medial wall of thalami can be clearly seen. $3 v$, III ventricle; Fx, fornix; ai, adesio interthalamica; Th, thalamus.

right); (2) an analysis of covariance with age as a covariate to control for the age effect on fornix; (3) an unpaired $t$ test on the corpus callosum volumes comparing nmTBI with the control group; (4) multiple linear regression analysis to measure predictability of the length of coma, estimated by using as dependent variables the volumes of the left and right hippocampus and the corpus callosum. For the dependent variable volume of the fornix, age was also included as a covariate; (5) Pearson partial correlations, used to search for a relationship between the neuropsychological test scores and the anatomical volumes. For the correlation with the fornix volume, there was no correction for age as the neuropsychological test scores are already corrected for age and education. In order to avoid type II error, $\mathrm{p}<0.05$ was considered significant without correction for the multiple tests. Without such a correction, higher sensitivity is obtained, but some of the significant brain-behaviour relationships could be false positives. Exact p values are reported in order to allow a direct evaluation of significance.
SPSS for Windows (release 10.0.1, SPSS Inc) was used to perform the statistical analyses.

\section{RESULTS}

\section{Gross morphological findings}

Hippocampus

The morphology of the hippocampi as visualised from the axial view of each studied brain is presented in fig 1 . In most of the nmTBIs, the hippocampal volume reduction was evident when compared with matched controls. Visual inspection of this figure does not allow detection of volume reduction in a specific location of the hippocampus. Furthermore, the shape of the hippocampus does not seem to be modified, but rather appears reduced overall.

\section{Fornix}

Inspection of fig 2 suggests that the size of the fornix in the severe nmTBI group is consistently reduced compared with 

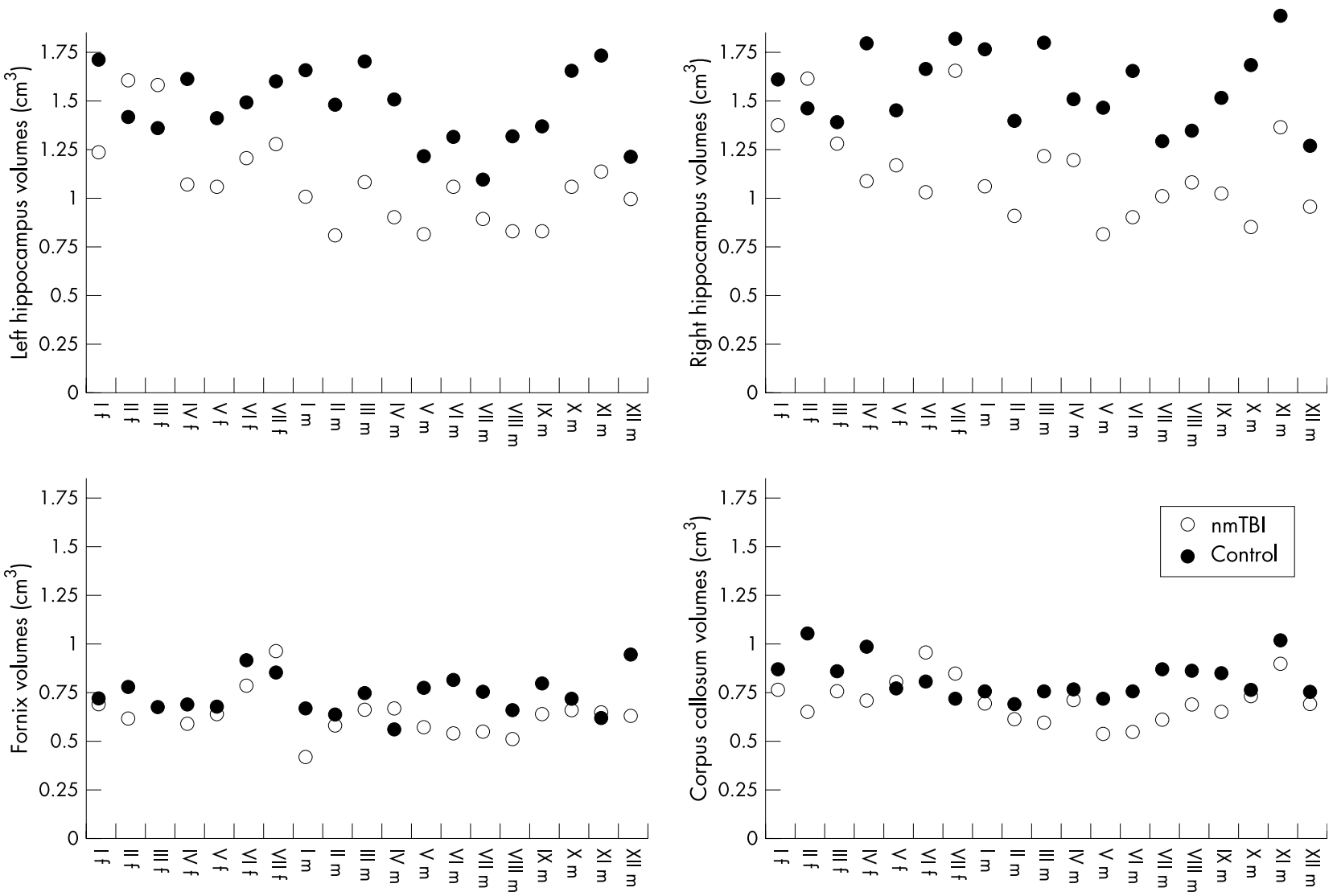

Figure 3 Volume measurements for each participant: left hippocampus, right hippocampus, fornix, and corpus callosum regions are normalised into the Talairach stereotaxic space. The unfilled symbols identify the nmTBI subject, and the filled symbols the normal control (C). The abscissa shows the ordinal numbers indicating the $\mathrm{nmTBI}$ and $\mathrm{C}$ subjects matched for gender and comparable for age. The dotted lines in the graphs represent the mean for each ROI. The inter-group comparison was significantly different for each ROI in that nmTBI had smaller volume than C.

gender and age matched control subjects, while the shape does not seem to be have been modified.

\section{Corpus callosum}

The morphology of the corpus callosum as visualised from the sagittal view of each brain is presented in fig 2 . Inspection of this figure suggests that the size of the corpus callosum in

Table 3 Correlations between cognitive tests and neuroanatomic measures

\begin{tabular}{|c|c|c|c|c|}
\hline Test & $\begin{array}{l}\text { Left } \\
\text { hipp }\end{array}$ & $\begin{array}{l}\text { Right } \\
\text { hipp }\end{array}$ & Fornix & $\begin{array}{l}\text { Corpus } \\
\text { callosum }\end{array}$ \\
\hline \multicolumn{5}{|c|}{ Words immediate recall $(n=19)$} \\
\hline$r$ & 0.09 & 0.11 & 0.58 & 0.50 \\
\hline$p$ & 0.70 & 0.64 & 0.01 & 0.03 \\
\hline \multicolumn{5}{|c|}{ Words delayed recall $(n=19)$} \\
\hline$r$ & 0.24 & 0.12 & 0.50 & 0.24 \\
\hline$p$ & 0.31 & 0.60 & 0.03 & 0.32 \\
\hline \multicolumn{5}{|c|}{ Short story immediate recall $(n=19)$} \\
\hline$r$ & 0.06 & -0.14 & 0.23 & 0.24 \\
\hline$p$ & 0.80 & 0.59 & 0.38 & 0.35 \\
\hline \multicolumn{5}{|c|}{ Short story delayed recall $(n=19)$} \\
\hline$r$ & 0.08 & -0.001 & 0.03 & -0.07 \\
\hline & 0.75 & 1.00 & 0.93 & 0.78 \\
\hline \multicolumn{5}{|c|}{ Rey figure immediate recall $(n=17)$} \\
\hline$r$ & 0.21 & 0.41 & 0.49 & 0.25 \\
\hline $\mathrm{p}$ & 0.42 & 0.10 & 0.05 & 0.33 \\
\hline \multicolumn{5}{|c|}{ Rey figure delayed recall $(n=16)$} \\
\hline $\mathrm{r}$ & 0.44 & 0.50 & 0.59 & 0.37 \\
\hline $\mathrm{p}$ & 0.09 & 0.05 & 0.02 & 0.16 \\
\hline
\end{tabular}

the severe nmTBI group was reduced in comparison with that of their matched control group. The shape of the corpus callosum in the nmTBIs also differs from that of the control subjects in that it appears to be more concave. Furthermore, an enlargement of the III ventricle in the nmTBI patients is evident, probably as consequence of the transneuronal degeneration of the thalamus ${ }^{36}$ that may produce part of the III ventricle enlargement. The most medial sagittal MRI section shows only the adesio interthalamica in the nmTBI patients, whereas in control subjects the medial wall of thalami can be clearly seen.

\section{Morphometric findings}

The volume of the ROIs in each investigated participant is plotted in fig 3.

\section{Hippocampus}

The main factor group was significant $\left(\mathrm{F}_{(1,36)}=40.078\right.$, $\mathrm{p}<0.001$ ), showing that the nmTBI group had on average smaller hippocampi than the control group (mean (SD) nmTBI 1.100 (0.232); normal control 1.512 (0.197)). Regardless of group, a significant difference $\left(F_{(1,36)}=12.314, p=0.001\right)$ was found between hippocampi, the right hippocampus $($ mean $=1.349$, sd. $=0.307)$ being larger than the left (mean (SD) 1.264, (0.286)). Similar differences have been previously reported..$^{34}$ The interaction was not significant.

\section{Fornix}

Age was significant $\left(\mathrm{F}_{(1,35)}=17.40, \mathrm{p}<0.001\right)$, indicating that, in our sample, fornix volume increased with age. 
However, controlling for age, the inter-group volume comparison revealed that the nmTBI group had a mean (SD) smaller fornix $(0.633(0.114))$ than the normal control group $(0.738(0.101))\left(F_{(1,35)}=10.80, p=0.002\right)$.

\section{Corpus callosum}

The between group comparison revealed that the nmTBI group had a smaller mean (SD) corpus callosum (0.699 $(0.111))$ than the normal control group $(0.814(0.103))$ $\left(\mathrm{t}_{(36)}=-3.264, \mathrm{p}<0.002\right)$.

\section{Predictability of the length of coma}

The length of coma was predictive of callosal volume reduction $(\mathrm{R}=-0.600, \mathrm{p}=0.007)$. The mean reduction of callosal volume was $3.863 \mathrm{~mm}^{3}$ for each day of coma. Neither the hippocampal volumes nor those of the fornix were predictable from the length of coma.

\section{Partial correlations between cognitive tests and ROI volumes}

The Pearson correlation results are summarised in table 3.

The volume of the right hippocampus correlated with the Rey figure delayed recall and the volume of the fornix correlated with immediate and delayed recall of both the Rey figure and the word list. Finally, the corpus callosum volume correlated with immediate recall of the word list. Immediate and delayed recall of the short story did not correlate with any of the neuroanatomical volumes examined.

\section{DISCUSSION}

This study evaluated changes in the gross morphology and in the volume of the hippocampi, fornix, and corpus callosum in patients with severe nmTBI. The patients sustained the head injury at least 90 days before data collection, a period during which some post-traumatic pathological changes such as wallerian degeneration ${ }^{38}$ and secondary axotomy ${ }^{39}$ occur and the transneuronal degeneration starts to appear. ${ }^{36}$ In the hippocampus, however, there is evidence of ongoing loss of neurones more than 6 months after injury. ${ }^{35}$

To estimate the volume modifications of specific anatomical structures and their relationship with behaviour and performance on neuropsychological tests without the confounding effect induced by large brain lesions, nmTBI patients with lesions that were larger overall than $1.6 \mathrm{~cm}^{3}$ were excluded from this study. As the neuropsychological assessment required co-operation and intact language functions, patients with severe language or attention deficits were also excluded from the study.

In order to reduce inter-individual variability in brain size, the brains were transformed into the Talairach proportional stereotaxic space. This transformation scaling adjusts (normalises) for all the brain structures allowing a direct intersubject comparison. It is a better normalisation procedure than others available because it protects from the noise introduced by a ratio ${ }^{17}{ }^{18}$ or by using the brain volume as a covariate in the statistical analysis. ${ }^{17} 1821$ As can be seen in fig 1 , rotation of the MRI brain volume along the main axis of the hippocampus provides a better visualisation of this anatomical structure and frequently permits detection of hippocampal volume reduction in nmTBI patients when comparing with control subjects. The hippocampal shape does not seem to be modified in any specific part, but rather appears to be reduced overall. However, in single case observations, if the volume reduction is not large or a clear asymmetry is not present, it remains difficult to recognise the hippocampal volume reduction. Only the group comparison makes it clear that a volume reduction of the hippocampi occurred in nmTBI patients.
As pointed out in the Methods section, an excellent visualisation of the corpus callosum in the sagittal plane can be obtained when the septum pellucidum and at least a large part of the falx are visible. In this view, the corpus callosum of the nmTBIs frequently appears reduced in volume compared with that of matched control subjects and its shape differs, in that it appears to be more concave. The group analysis of the volumetric measurements confirmed the impression derived from the visual inspection that the nmTBI group had a smaller corpus callosum compared with the control group. Similar findings had been reported previously. ${ }^{10-12}$ A reduction in the volume of the fornix in the nmTBI group was also observed. This result is in keeping with earlier reports based on two dimensional MRI fornix measurement. ${ }^{8-10}$ Both fornix and corpus callosum reductions in patienents with nmTBI are most likely the manifestation of diffuse axonal damage.

The length of coma in the present study was predictive of the reduction in callosal volume, consistent with some previous evidence of such a relationship. ${ }^{1-13}$ Considering that volume reduction in the corpus callosum is the result of diffuse axonal damage, our results are in keeping with neuropathological data on closed head injury in monkeys ${ }^{40}$ that revealed a correlation between the length of coma and the extent of the diffuse axonal damage.

The nmTBI patients in our sample had memory impairment that was disproportionate to their general cognitive deficit. As it can be seen in table 2, with the exception of two patients, the Wechsler IQ in these patients was consistently higher than their memory quotient, the discrepancy ranging from 3 to 25 points. ${ }^{41}$ The memory impairment extended to both verbal and visual-spatial information. Indeed, each patient of the nmTBI group performed poorly on at least one of the six memory tests and with the exception of two cases, one scoring poorly only on the verbal tests, the other performing pathologically only on the visual-spatial tests, the impairment was pervasive, involving poor recall of both verbal and visual-spatial data.

The results of the correlational analysis weakly support the hypothesis of a relationship between degree of volume reduction of the neural structures examined and severity of the memory impairment. Indeed, of 24 correlation coefficients calculated between the 4 anatomical volumes examined and the performance on the 6 memory tests administered, 21 were in the expected direction (worse performance scores as the volume decreased). However, only in a minority of cases $(6 / 24)$ did these correlations reached the conventional level of statistical significance. It has to be considered that some of the significant brain-behaviour relationships could be false positives, as mentioned in the Methods section.

Unlike earlier studies, which generally failed to provide evidence of an association between fornix volume reduction and performance on memory tests,,$^{5-10}$ the present study showed that the volume of the fornix had the most consistent pattern of association with memory scores (see table 3 ). Perhaps, with respect to a previous study that measured the fornix area in a single axial slice, ${ }^{8}$ the method used in the present study to obtain the fornix volume from multiple measurements in consecutive axial slices may have provided a more sensitive estimate of the degree of fornix volume reduction.

The volume of the right hippocampus correlated significantly with delayed reproduction of the Rey figure, consistent with the role of this anatomical structure in visual-spatial memory. ${ }^{42}$ Finally, the corpus callosum volume correlated with performance in the immediate word list recall task. Memory of the short story did not correlate with any of the anatomical measures; however, only a few nmTBI patients 
scored poorly on this test. It could be speculated that poor sensitivity of this task might be one of the possible reasons for this lack of correlation.

Overall, the extent of volumetric reduction in the hippocampus, fornix, and corpus callosum accounted for only a limited portion of the variance in the memory scores of patients with nmTBI. In particular, although neuropsychological evaluation in amnesic patients with hippocampal damage $^{43}$ and/or atrophy ${ }^{44}$ points to a significant role of the hippocampal formation in prose recall, we did not find any correlation between hippocampal and fornix volumes and short story recall. There could be at least three, not exclusive, reasons for this observation. (a) As regards the hippocampus, it should be noted that the measurement in the present study involved only the dentate gyrus and cornu ammonis. Data from both the animal and human literature suggest that the memory impairment may be more evident in cases with more extensive damage to the medial temporal region including the subiculum and entorhinal cortex ${ }^{45-47}$ Had we obtained a volume of the entire mesial temporal region, a more consistent pattern of association between hippocampal region volume reduction and memory deficit might have emerged. (b) A statistical control for the continuous hippocampal gray matter loss in patients with nmTBI has been used assuming a linear reduction. However, this process may not be linear but rather "per stage" as has been observed in hippocampal neural apoptosis. ${ }^{48}$ In addition, it is possible that in this study the volumetric measures were obtained while the neural loss was still in progress, even though the cognitive impairment might have stabilised. A follow up study investigating the brain MRI of the same patients across different periods of time from injury may address the macroscopic brain modification of the nmTBI and its possible correlation with behaviour. Moreover, it has to be stated that although corpus callosum and fornix may represent some indicators of the non-specific nature of brain injury, there may be focal white matter lesions-that is, undetected white matter pathology, disseminated in the brain of the severe nmTBI patients that may be locally disruptive to function. (c)The assumption of a close relationship between memory deficit and damage in a single neural structure inpatients with nmTBI is likely to be an oversimplification. The diffuse nature of the brain damage in these patients probably affects the neural circuitry implicated in information processing, memory storage and retrieval at multiple levels, thus resulting in a complex and multivariate relationship between brain lesion/s and memory performance.

In conclusion, the present study demonstrated that a volume reduction in hippocampus, fornix, and corpus callosum is detectable in nmTBI, even when no detectable macroscopic lesions are observed. Improved morphological investigation of the corpus callosum and the hippocampus can be obtained by using the particular MRI brain orientations suggested in the present study, but quantitative group measures are needed to ensure a correct estimation of volume reduction in these structures. The duration of the coma is predictive of the corpus callosum volume and thus of the overall white matter damage. Finally, of the several measures of memory test performance examined, only a few correlated with volumetric reduction. We believe that the lack of correlation may reflect the diffuse nature of the damage and/or progressive neuronal loss happening a considerable period of time after injury. To address the macroscopic brain modification of the nmTBI and the possible correlation with behaviour, a follow up study investigating the nmTBI brain of the same subject across different periods of time from injury is needed. The volumetric measures obtained while the neural loss is still in progress should provide better information about the anatomical and behavioural modifications of this likely progressive brain pathology.

\section{ACKNOWLEDGEMENTS}

Special thanks to Drs J D MacDonald and A C Evans of the Montreal Neurological Institute, McGill University, Canada, for providing us with Display.

\section{Authors' affiliations}

F Tomaiuolo, G A Carlesimo, M Di Paola, R Bonanni, R Formisano,

C Caltagirone, IRCCS Fondazione 'Santa Lucia', Rome, Italy

G A Carlesimo, C Caltagirone, Clinica Neurologica, Rome University, Tor Vergata, Italy

M Petrides, Montreal Neurological Institute, McGill University, Montreal Quebec, Canada

F Fera, IMSEB - CNR Cosenza, Italy

P Pasqualetti, AFaR Ospedale 'Fatebenefratelli' Roma, Italy

Competing interests: none declared

\section{REFERENCES}

1 Adams $\mathrm{JH}$. Brain damage in fatal non-missile head injury in man. In: Braakman R, eds. Handbook of clinical neurology: head injury. Amsterdam Elsevier Science Publishers BV, 1990;13:43-63.

2 Wilson JTL. Significance of MRI in Clarifying whether neuropsychological deficits after head injury are organically based. Neuropsychology 1990;4:261-9.

3 Azouvi P. Neuroimaging correlates of cognitive and functional outcome after traumatic brain injury. Curr Opin Neurol 2000;13:665-9.

4 Bigler, ed. Quantitative magnetic resonance imaging in traumatic brain injury. $J$ Head Trauma Rehabil 2001;16:117-34.

5 Bigler ED Blatter DD, Gale SD, et al. Traumatic brain injury and memory: the role of hippocampal atrophy. Neuropsychology 1996;3:333-42.

6 Bigler ED, Blatter DD, Anderson CV, et al. Hippocampal volume in normal aging and traumatic brain injury. Am J Neuroradiol 1997; 18:11-23.

7 Bigler ED, Anderson CV, Blatter DD, et al. Temporal lobe morphology in normal aging and traumatic brain injury. Am J Neuroradiol 2002;23:255-66.

8 Tate DF, Bigler, eds. Fornix and hippocampal atrophy in traumatic brain injury. Learn Mem 2000;7:442-6.

9 Gale SD, Burr RB, Bigler ED, et al. Fornix degeneration and memory in traumatic brain injury. Brain Res Bull 1993;32:345-9.

10 Gale SD, Johnson SC, Bigler ED, et al. Nonspecific white matter degeneration following traumatic brain injury. J Int Neuropsychol Soc 1995;1:17-28.

11 Levin HS, Williams DH, Valastro M, et al. Corpus callosal atrophy following closed head injury: detection with magnetic resonance imaging. J Neurosurg 1990:73:77-81.

12 Henry-Feugeas MC, Azouvi P, Fontaine A, et al. MRI analysis of brain atrophy after severe closed-head injury: relation to clinical status. Brain $\ln i$ 2000;14:597-604.

13 Azouvi P, Hurtier O, Tougeron A, et al. Imagerie par resonance magnetique et sequelles neurologiques des traumatismes craniens graves. Annales de Readaptation et de Medicine Physique 1993;36:1-11.

14 Capruso DX, Levin HS. Cognitive impairment following closed head injury. Neurol Clin 1992;10:879-93.

15 Wilson JT, Hadley DM, Wiedmann KD, et al. Neuropsychological consequences of two patterns of brain damage shown by MRI in survivors of severe head injury. J Neurol Neurosurg Psychiatry 1995;59:328-31.

16 Wallesch CW, Curio N, Galazky I, et al. The neuropsychology of blunt head injury in the early postacute stage: effects of focal lesions and diffuse axonal injury. J Neurotrauma $2001 ; 18: 11-20$.

17 Jancke L, Preis S, Steinmetz H. The relation between forebrain volume and midsagittal size of the corpus callosum in children. Neuroreport $1999 ; 10: 2981-5$

18 Jancke L, Staiger JF, Schlaug G, et al. The relationship between corpus callosum size and forebrain volume. Cereb Cortex 1997;7:48-56.

19 Witelson SF. Hand and sex differences in the isthmus and genu of the human corpus callosum. A postmortem morphological study. Brain $1989 ; 112: 799-835$.

20 Schmitt JE, Eliez S, Warsofsky IS, et al. Corpus callosum morphology of Williams syndrome: relation to genetics and behavior. Dev Med Child Neurol 2001;43:155-9.

21 Bermudez P, Zatorre RJ. Sexual dimorphism in the corpus callosum: methodological considerations in MRI morphometry. Neuroimage 2001;13:1121-30.

22 Collins DL, Neelin P, Peters TM, et al. Automatic 3D intersubject registration of MR volumetric data in standardized Talairach space. J Comput Assist Tomogr 1994; 18:192-205.

23 Paus T, Otaky N, Caramanos Z, et al. In vivo morphometry of the intrasulcal gray matter in the human cingulate, paracingulate, and superior-rostral sulci: hemispheric asymmetries, gender differences and probability maps. J Comp Neurol 1996;23:664-73.

24 Penhune VB, Zatorre RJ, MacDonald JD, et al. Interhemispheric anatomical differences in human primary auditory cortex: probabilistic mapping and 
volume measurement from magnetic resonance scans. Cereb Cortex 1996:6:661-72.

25 Tomaivolo F, MacDonald JD, Caramanos Z, et al. 1999 Morphology, morphometry and probability mapping of the pars opercularis of the inferior frontal gyrus: an in vivo MRI analysis. Eur J Neurosci 1999:11:3033-46.

26 Tomaiuolo F, Di Paola M, Caravale B, et al. Morphology and morphometry of the corpus callosum in Williams syndrome: A T1-weighted MRI study. Neuroreport 2002;3:2281-4

27 Wechsler D. Wechsler adult intelligence scale-revised. San Antonio, TX: The Psychological Corporation, 1981.

28 Wechsler DA. Scala di intelligenza per adulti. Firenze: Organizzazioni Speciali, 1981.

29 Capitani E. Metodi statistici. In: Spinnler H, Tognoni G, eds. Standardizzazione e Taratura Italiana di test psicometrici ltal. J Neurol Sci 1987; (Suppl 8):14-20

30 Carlesimo GA, Caltagirone C, Gainotti G. The Mental Deterioration Battery normative data, diagnostic reliability and qualitative analysis of cognitive impairment. The Group for the Standardization of the Mental Deterioration Battery. Eur Neurol 1996;36:378-84.

31 Carlesimo GA, Buccione I, Fadda L, et al. Standardizzazione di due test di memoria: Breve Racconto e Figura di Rey. Nuova Rivista di Neurologia 2002;12:1-13

32 Rey A. Reattivo della figura complessa. Manuale. (Complex figure task. Manual). Firenze: Organizzazioni Speciali, 1968.

33 Salat D, Ward A, Kaye JA, et al. Sex differences in the corpus callosum with aging.

34 Pruessner JC, Li LM, Serles W, et al. Volumetry of hippocampus and amygdala with high-resolution MRI and three-dimensional analysis software: minimizing the discrepancies between laboratories. Cereb Cortex 2000;10:433-42.

35 Maxwell WL, Dhillon K, Harper $\mathrm{L}$, et al. There is differential loss of pyramidal cells from the human hippocampus with survival after blunt head injury. J Neuropathol Exp Neurol 2003;62:272-279
36 Adams JH, Graham DI, Jennett B. The neuropathology of the vegetative state after an acute brain insult. Brain 2000;123:1327-38

37 Watson C, Andermann F, Gloor P, et al. Anatomic basis of amygdaloid and hippocampal volume measurement by magnetic resonance imaging. Neurology 1992;42:1743-50.

38 Adams JH, Graham DI. An introduction to neuropathology, 2nd ed. Edinburgh: Churchill Livingstone, 1994.

39 Maxwell WL, Povlishock JT, Graham DL. A mechanistic analysis of nondisruptive axonal injury: a review. J Neurotrauma 1997;14:419-40.

40 Gennarelli TA, Thibault LE, Adams JH, et al. Diffuse axonal injury and traumatic coma in the primate. Ann Neurol 1982;12:564-74.

41 Levin HS, Goldstein FC, High WM Jr, et al. Disproportionately severe memory deficit in relation to normal intellectual functioning after closed head injury. J Neurol Neurosurg Psychiatry 1988;10:1294-301.

42 Milner B. Visulal recognition and recall after right temporal lobe lesion in man. Neuropsychol 1968;6:191-209.

43 Spiers HJ, Maguire EA, Burgess N. Hippocampal amnesia. Neurocase $2001 ; 7: 357-82$.

44 Kilpatrick C, Murrie V, Cook M, et al. Degree of left hippocampal atrophy correlates with severity of neuropsychological deficits. Seizure 1997;6:213-18.

45 Scoville, Milner B. Loss of recent memory after bilateral hippocampal lesions. J Neuropsychiatry Clin Neurosci 1957;20:11-21.

46 Mishkin M. Memory in monkeys severely impaired by combined but not by separate removal of amygdala and hippocampus. Nature 1978;273:297-8

47 Squire LR, Zola-Morgan S. The medial temporal lobe memory system. Science 1991;20:1380-6.

48 Runnerstam M, Bao F, Huang Y, et al. A new model for diffuse brain injury by rotational acceleration: Il. Effects on extracellular glutamate, intracranial pressure, and neuronal apoptosis. I Neurotrauma $2001 ; 18: 259-73$ 